\title{
Performance of MV Distributed Energy Power Systems under Model-Predictive Control and Conventional Power Systems State Estimators
}

\author{
John Sandoval-Moreno \\ École Polytechnique (chaire TOTAL) \\ Palaiseau, France \\ Email: john-anderson.sandoval-moreno@polytechnique.edu
}

\author{
Carlos Ocampo-Martinez, IEEE Senior Member \\ Universitat Politècnica de Catalunya \\ Institut de Robòtica i Informàtica Industrial, CSCI-UPC \\ Barcelona, Spain \\ Email: cocampo@iri.upc.edu
}

\begin{abstract}
In this paper, the performance of a middle voltage (MV) distributed power generation system under a Model Predictive Control (MPC) strategy is tested. The designed controller receives process information from an state estimation unit, composed of a Weighted Least-Squares strategy (WLS). The contribution is focused on performing the frequency and voltage regulation of the system, considering moreover the dynamic behavior of storage units and capacitors, and feeding back information to the control system considering the transmission delay. The results show that the closed loop based on the proposed strategy achieves the required performance and can be useful to be applied to more complex systems such as low-voltage (LV) generation systems.
\end{abstract}

\section{INTRODUCTION}

In the control of distributed power generation systems, there are typically multiple objectives to be achieved, according to the control hierarchy that is considered [1]. In this sense, power system stability is always required, which is represented by respecting the frequency and voltage magnitude constraints. Both of them can be manipulated through the active and reactive power generated at the power system nodes, respectively [1], [2].

However, control structures such as the Model Predictive Control (MPC) that is here used and has been applied in power system control solutions [3], [4], [5], [6], always demand information from the power system in form of measurements using expensive sensors or using estimated measurements through the results of distribution system state estimators [7], [8] supported by the load-flow computations of the system [1].

Even if this information is available and the estimation is precise, the delay between the estimated values and the control system, which is always performing at real time, should be considered, for instance, in the controller structure. The MPC structure here considered [3], [4] takes into account that disturbances are taken as static values until an external estimation block updates them. Of course, the controller maintains the regulation, considering the system constraints and adjusting the control signals accordingly. Having in mind this feature of the MPC, in this paper the performance of a middlevoltage (MV) distributed power system is evaluated, which is characterized by five generation areas, each of them with a synchronous generator, an energy storage element and a renewable-based power production, this latter interacting with the system through an inverter and considered at optimal power production.

For the state estimation, the system has sensors only in the generation nodes as form of power injections and voltage magnitudes $\left(P_{i}, Q_{i},\left|V_{i}\right|\right)$. The other information, composed of no measurable loads and null injections will be given as pseudomeasurements and virtual measurements that are associated with larger and smaller variances in the real measurements [7], [8]. The information will be processed according to the controller requirements, but in different sampling rate, compared to that used for the discrete-time control structure. The idea is to evaluate how the state estimation period affects the system performance in this controller structure.

The remainder of this paper is organized as follows. Section II introduces the power network topology and the structure of each generation area (microgrid) considered, Section III describes the state estimation algorithm and the MPC structure proposed, Section IV presents some simulation results of the proposed scheme and finally, Section V collects and discusses some conclusions and perspectives of this work.

\section{Notation}

Along this paper, the following notation is stated for the dynamic system modelling: vectors $x, u$ and $d$ represent the states, inputs and disturbance vectors, with super indexes $\sim$ and $*$, and sub-indexes 0 and $s$ indicating deviation, reference, initial and static value, respectively. Matrices $A, B$ and $E$ are the dynamical, inputs and disturbance matrices.

For the power system state estimation, $z$ represents the estimated vector, which is composed of the voltage magnitudes and phase angles for each node $i=1 \cdots n$ as $z=$ $\left[\left|V_{1}\right|\left|V_{2}\right| \cdots\left|V_{n}\right| \theta_{1} \theta_{2} \cdots \theta_{n}\right], h_{m}(z)$ and $\sigma_{m}$ for $m=1 \cdots r$ are the measurements functions and the standard deviation of each one of them and $\mu_{m}$ are the measured quantity, while, $\hat{z}$ represents the estimated states vector.

In general, $P_{i}, Q_{i}, P_{i j}, Q_{i j}, P_{L, i}, Q_{L, i}, P_{g, i}, Q_{g, i}$ represent the active and reactive power injections, power line flows between nodes $i$ and $j$, power loads and generated power, 
respectively. If the index $\widehat{ }$ appears with the quantity, it is assumed that a load flow calculation was done using $\hat{z}[1]$, [7].

\section{Power System Structure And Local MICROGRIDS MODELING}

\section{A. Network Characteristics}

In Figure 1, it is shown the power network topology, which is based in the IEEE 13-buses radial test feeder [9]. For this paper, it is considered a system with five generation areas at nodes $1,2,6,8$ and 12 , along with 8 loaded nodes at $1,2,6,7,8,12,13$ and 14 . The resistance, inductive reactance and capacitive susceptance in per unit, are shown in Table I. The power system capacity is $1 M V A / 13.2 k V A$. Moreover, a three-phase and balanced power system is considered.

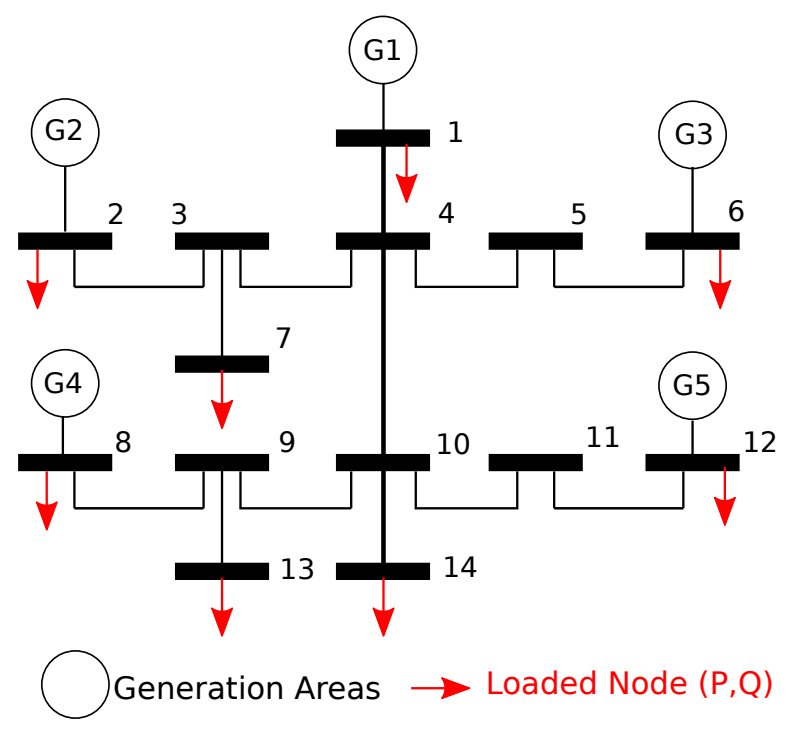

Fig. 1. Power system structure

TABLE I

POWER LINES SPECIFICATIONS (IN P.U)

\begin{tabular}{c|c|c|c|c|c|c|c} 
Line & $\mathbf{R}$ & $\mathbf{X}$ & $\mathbf{B}$ & Line & $\mathbf{R}$ & $\mathbf{X}$ & $\mathbf{B}$ \\
\hline $1-4$ & 0.155 & 0.44 & $2 \mathrm{e}-5$ & $8-9$ & 0.05 & 0.078 & $1 \mathrm{e}-5$ \\
\hline $2-3$ & 0.087 & 0.089 & $1 \mathrm{e}-5$ & $9-10$ & 0.087 & 0.089 & $1 \mathrm{e}-5$ \\
\hline $3-4$ & 0.087 & 0.049 & $7 \mathrm{e}-4$ & $10-11$ & 0.015 & 0.006 & $1 \mathrm{e}-4$ \\
\hline $4-5$ & 0.084 & 0.13 & $1 \mathrm{e}-5$ & $11-12$ & 0.087 & 0.049 & $7 \mathrm{e}-4$ \\
\hline $5-6$ & 0.029 & 0.030 & $1 \mathrm{e}-5$ & $9-13$ & 0.235 & 0.090 & $2 \mathrm{e}-4$ \\
\hline $3-7$ & 0.235 & 0.090 & $10 \mathrm{e}-4$ & $10-14$ & 0.008 & 0.022 & $1 \mathrm{e}-5$ \\
\hline $4-10$ & 0.152 & 0.445 & $2 \mathrm{e}-4$ & & & & \\
\hline
\end{tabular}

\section{B. Generation Areas Description and Discrete-Time Model}

Every generation area is composed, as showing in Figure 2, by a hybrid microgrid in which synchronous generators, a battery and renewable power input are included. The renewable power input is not controlled and considered optimal from an external algorithm.

The proposed mathematical model represents the system dynamics from the point of view of power flows rather than current flows. Therefore, the synchronous generator is modeled considering a simplified model of the governor and turbine as proposed in [1], [2], [3], [4]. The battery converter is considered as a pure active-power bidirectional unit, while the block that includes the renewable power source is modeled as suggested by Yazdani et al [10], which also includes the output filter capacities for delivering reactive power, but considering a decoupled behavior between active and reactive power control generation [10]. For each block (generator, battery, renewable

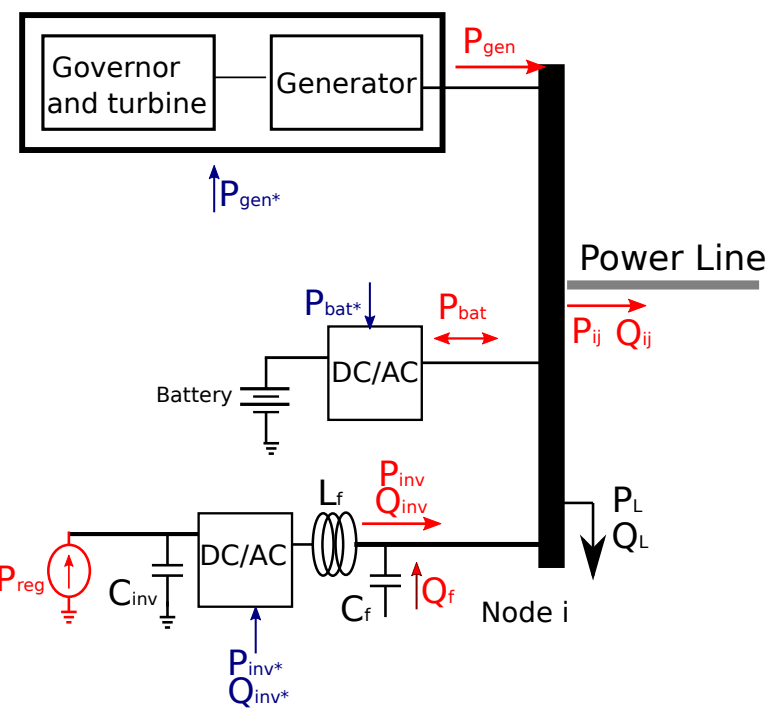

Fig. 2. Structure of the hybrid microgrids

source), the small-signal discrete-time model is obtained, taking into account the sampling period $T_{s}$ and the following signals:

$$
\begin{aligned}
& x=\left[\begin{array}{lllll}
\theta & f & S o C & v_{i n v} & \delta q_{i n v}
\end{array}\right]=\left[\begin{array}{llll}
x_{1} & x_{2} & x_{3} & x_{4}
\end{array}\right],
\end{aligned}
$$

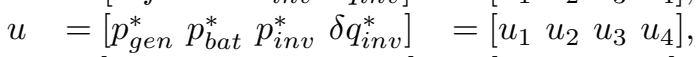

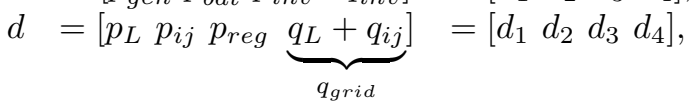

where $\theta$ is the voltage phase angle equal to the rotatory machine angle, $f$ is the line frequency, $S o C$ is the stateof-charge of the batteries, $v_{i n v}$ is the voltage of the DC capacitor at the renewable source inverter, $\delta q_{i n v}$ is the reactive power generated by the renewable source inverter, $p_{g e n}^{*} p_{b a t}^{*} p_{i n v}^{*}$ and $\delta q_{i n v}^{*}$ are the references of active power from the synchronous generator, battery, renewable inverter and reactive power renewable inverter, respectively, while $p_{L} p_{i j} p_{\text {reg }}$ and $q_{g r i d}$ are the active load, active power flow, active power from the renewable source and reactive power at the grid, which is the sum of the reactive power load and reactive power line flow. 
1) Synchronous Generator Model: Considering simplified dynamics, the following equations describe the unit [4]:

$$
\begin{gathered}
x_{1}^{k+1}=x_{1}^{k}+2 \pi T_{s} x_{2}^{k}, \\
x_{2}^{k+1}=\left\{\begin{array}{l}
\left.1-\frac{a T_{s}}{T_{N}}\right) x_{2}^{k}+ \\
\left(\frac{T_{s}}{T_{N}}\right) \cdot\left[b u_{1}^{k}+b u_{2}^{k}-c d_{1}^{k}+c d_{2}^{k}-c d_{3}^{k}\right] .
\end{array}\right.
\end{gathered}
$$

Constants $a, b$ and $c$ are related with gains and time constants associated to the generator and an internal frequency-droop strategy [1].

2) Battery Model: The battery model represents only the state-of-charge variations with respect to the battery power (i.e., capacitive model simplification) as follows:

$$
x_{3}^{k+1}=x_{3}^{k}-T_{s} K_{\text {soc }} u_{2}^{k},
$$

where $K_{s o c}$ is a constant associated with the battery capacity.

3) Renewable Generator Inverter: This unit was modelled according to the recommendation found at [10], where it is also highlighted that any interaction between active and reactive power control can be neglected when internal current loops are included in the structure. For this case, these dynamics are simplified and only power flows are taken into account. However, in this structure two elements are considered: the DC/AC input capacitor that should maintain the voltage accordingly for a correct functionality of the converter, and the reactive power generation, which is assumed for this unit. Here, the required reactive power from the inverter is the difference between the grid power (the sum of reactive load and reactive power line flow) minus the power supplied by the capacitor $C_{f}$ [10]. In this sense, the following expressions for the DC capacitor and the reactive power dynamics can be proposed:

$$
\begin{aligned}
x_{4}^{k+1} & =x_{4}^{k}+\frac{T_{s}}{K_{D C}}\left(d_{3}^{k}-u_{3}^{k}\right), \\
x_{5}^{k+1} & =\left(1-\frac{T_{s}}{T_{Q}}\right) x_{5}^{k}+\frac{K_{Q} T_{s}}{T_{Q}}\left(d_{4}^{k}+u_{4}^{k}\right) .
\end{aligned}
$$

In this structure, the reactive power production includes the disturbance knowledge (reactive grid power for the node) and the power demand reference is compensated by $u_{4}$. Parameters $K_{D C}, T_{Q}$ and $K_{Q}$ are associated with input capacitor capacitance, time constant and gain of the converter for the reactive power compensation, respectively.

For every microgrid, the parameter names are equivalent. Considering this, the parameter values in vector notation are shown in Table II.

\section{Distribution System State Estimation And Model Predictive Control Structure}

\section{A. State Estimator Structure Overview}

The state estimation structure is designed to obtain precise

\begin{tabular}{|c|c|c|c|}
\hline Parameter & Value $\left(G_{1} \cdots G_{5}\right)$ & Parameter & Value $\left(G_{1} \cdots G_{5}\right)$ \\
\hline $\mathbf{a}$ & 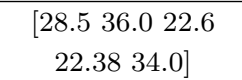 & b & $\begin{array}{c}{\left[\begin{array}{llll}110 & 100 & 108 \\
85.5 & 95.0\end{array}\right]}\end{array}$ \\
\hline c & $\begin{array}{c}{\left[\begin{array}{ccc}110 & 100 & 120 \\
90 & 100\end{array}\right]}\end{array}$ & $T_{N}$ & $\begin{array}{c}{\left[\begin{array}{lll}25 & 18 & 20 \\
25 & 22\end{array}\right]}\end{array}$ \\
\hline$K_{s o c}$ & 50 for all systems & $K_{D C}$ & 83 for all systems \\
\hline$T_{Q}$ & 0.50 for all systems & $K_{Q}$ & 0.225 for all systems \\
\hline
\end{tabular}
knowledge from the power network (e.g., nodal voltage magnitude and phase, nodal active and reactive power injections,
TABLE II

PARAMETERS FOR EACH MICROGRID SYSTEM

active and reactive power flows), from the available measurements $\mu_{m}=1 \cdots r$ that can be real measurements (sensors), pseudo measurements (predictions) and virtual measurements (associated moreover with null power injections).

The selection of the states vector depends exclusively on the design and requirements of the control system [7] [11], and the associated errors will define the variance of each measurement. Typically, the percentage of errors belongs to the range $0.5-1 \%$ for real measurements, $30-80 \%$ for the pseudo measurements and $<0.01 \%$ for the virtual measurements [1][7]. The state estimator in this paper focuses on obtaining a vector that represents both, the magnitude and phase of the voltage phasors at each node, $z=\left[\left|V_{1}\right|\left|V_{2}\right| \cdots\left|V_{n}\right| \theta_{1} \theta_{2} \cdots \theta_{n}\right]$ by solving the following optimization problem:

$$
\min \frac{1}{2} \sum_{m=1}^{r}\left(\mu_{m}-h_{m}(z)\right)^{T} W_{m m}\left(\mu_{m}-h_{m}(z)\right),
$$

where $W_{m m}=1 / \sigma_{m}^{2}$ is a weight associated to each measurement $\mu_{m}$, with a measurement error distributed in a normal distribution of mean $\mu_{m}$ and variance defined as follows, computed at every state estimation [7]:

$$
\sigma_{m}^{2}=\left(\frac{\mu_{m} \epsilon_{m}}{300}\right)^{2},
$$

with $\epsilon_{m}$ representing the precision of the measurements of $\mu_{m}$ ( $1 \%$ for real measurements, $50 \%$ for pseudo measurements and $0.1 \%$ for virtual measurements). The factor 300 allows to include the $100 \%$ of values over the normal distribution [7]. The functions $h_{m}(z)$ depend on the kind of measurements, which are, in this paper, only voltage magnitude and active and reactive power injections expressed as follows [7]:

$$
\begin{aligned}
V_{i} & =\left|V_{i}\right|, \\
P_{i} & =\left|V_{i}\right| \sum\left|V_{j}\right|\left(g_{i j} \cos \left(\theta_{i}-\theta_{j}\right)+b_{i j} \sin \left(\theta_{i}-\theta_{j}\right)\right), \\
Q_{i} & =\left|V_{i}\right| \sum\left|V_{j}\right|\left(g_{i j} \sin \left(\theta_{i}-\theta_{j}\right)-b_{i j} \cos \left(\theta_{i}-\theta_{j}\right)\right),
\end{aligned}
$$

where $i$ represents the measured node, $j$ the adjacent nodes from $i$, while $b_{i j}$ and $g_{i} j$ are the line parameters for the $i-j$ line.

Problem (7) should be solved by using iterative methods. To do so and after some manipulation with respect to the minimization variable $z$, the following recursive expression are obtained in order to find the estimated vector $\hat{z}$ :

$$
\hat{z}_{l+1}=\hat{z}_{l}+G_{l}^{-1} H_{l}^{T} W\left(\bar{\mu}-h\left(\hat{z}_{l}\right)\right),
$$


where $H_{l}=\frac{\partial h_{m}}{\partial \hat{z}_{l}}$ is a Jacobian matrix that relates every measurements with the states vector and $G_{l}=H_{l}^{T} W H_{i}$ is a gain matrix. Moreover, the steps are characterized by the subindex $l$ and the iterative procedure stops when $\left|\hat{z}_{l+1}-\hat{z}_{l}\right| \leq t o l$. In (12), $\mu$ represents the measurements vector that is computed for each component as follows:

$$
\bar{\mu}_{m}=\mu_{m}\left(1+\frac{\epsilon_{m} \eta}{300}\right)
$$

where $\eta$ represents a random value with a normal distribution, which is assumed to represent the measurement errors and the factor 300 has the same meaning than in (8). In the state estimation, the measurement values should be kept constant during the interaction process that once finished, it delivers a estimated measurements vector $\hat{z}$ that will depend exclusively on the estimated states.

The state estimation can be delayed with respect to the current network state due to the computational time required for collecting and processing the information that, in practical distribution networks, can take from 1 to 10 minutes to be treated. In this paper, it is used a lower time scale $(10 \mathrm{~s})$, due to the power system structure and the computational performance of the estimator.

Finally, the state estimator is designed and stated to return some useful values from the process, considering that in real applications, to obtain measurements from all the nodes or lines can be quite expensive. Thus, the use of some real measurements and some predicted values, especially power loads, are taken in consideration for the reconstruction of the process signals and determination of the system dynamics.

\section{B. Model Predictive Controller}

Due to the lack of space, details about the MPC statement and design for the applications considered here are not included. The reader is addressed to [3][4]for further details. First of all, the system dynamics are described as follows:

$$
x_{k+1}=A x_{k}+B u_{k}+E d_{k},
$$

where $x \in \Re^{n}, u \in \Re^{m}, d \in \Re^{q}$ are the states, inputs and disturbances vectors, respectively, and matrices $A \in$ $\Re^{n x n}, B \in \Re^{n x m}, E \in \Re^{n x q}$ define the system dynamics in (14). The MPC controller delivers an input signal after solving the following optimization problem over a prediction horizon $N$ :

$$
\begin{array}{ll} 
& \min \frac{1}{2}\left(\tilde{x}_{N}^{T} P \tilde{x}_{N}+\sum_{k=0}^{N-1} \tilde{x}_{k}^{T} Q \tilde{x}_{k}+\tilde{u}_{k}^{T} R \tilde{u}_{k}\right) \\
\text { s.t } & x_{\min } \leq x_{k} \leq x_{\max }, \forall k=1 \ldots N \\
& u_{\min } \leq u_{k} \leq u_{\max }, \forall k=1 \ldots N \\
& x_{k+1}=A x_{k}+B u_{k}+E d_{k}, \forall k=1 \ldots N
\end{array}
$$

where $Q$ and $R$ are weighting matrices while $P$ is the solution of the discrete-time Ricatti Algebraic Equation for the system in (14) [3].

The sequences of control inputs and disturbances over $\mathrm{N}$ are defined as follows:

$$
\begin{aligned}
& \boldsymbol{u}=\left[\begin{array}{ll}
u_{1} & u_{2} \\
\cdots & u_{N}
\end{array}\right], \boldsymbol{u} \in \Re^{N . m} \\
& \boldsymbol{d}=\left[\begin{array}{ll}
d_{1} & d_{2} \cdots d_{N}
\end{array}\right], \boldsymbol{d} \in \Re^{N . q}
\end{aligned}
$$

Then, the problem (15) can be rewritten in a quadratic way, optimizing the sequence input vector $\boldsymbol{u}$ and considering the current state $x_{0}$ and the disturbance vector $\boldsymbol{d}$ that can be constant for every instant, that is:

$$
\begin{array}{ll}
\min & \frac{1}{2} \boldsymbol{u}^{T} H \boldsymbol{u}+\boldsymbol{u}^{T}\left[\boldsymbol{K}_{\mathbf{1}}\left(x_{0}-x_{s}\right)+\boldsymbol{K}_{\mathbf{2}}\left(\boldsymbol{d}-\boldsymbol{d}_{\boldsymbol{s}}\right)-H \boldsymbol{u}_{\boldsymbol{s}}\right] \\
\text { s.t } & L \boldsymbol{u} \leq W .
\end{array}
$$

Matrices $H, K_{1}, K_{2}, L$ and $W$ are function of the predicted dynamics and weighting matrices $Q$ and $R$, and their structure can be seen in [3], [4]. Therefore, if enough information about disturbances is available, the controller computes the optimal solution that respects the constraints and stabilizes the system.

\section{Decentralized structure and feedback information for the considered power system}

Having in consideration that each microgrid is connected to one line, it can be assumed that every area regulates the amount of exchanged power with the network, even if there are nodes not directly connected but still loaded. In this sense, having a look at Figure 2 and the microgrids model (1)-(6), it can be seen that the disturbances $p_{L}, p_{i j}$ and $q_{\text {grid }}$ are required for controlling the power generation for each generation area. Therefore, in every state estimation, which is programmed to be in a sampling time larger that the sampling period, a load flow calculation is done with the results from the state estimator. It is also assumed that the system states are known and well measured.

In this way and as indicated in [3], the disturbance vector will be at least partially known and retained as the true disturbances. Nevertheless, the MPC controller will assure the system performance, because states of interest such as the frequency, SoC, the capacitor voltage for the inverter and the correction for the reactive power generation will be assured locally, but using the information from the network in form of power flow from the generation nodes to the rest of the system. As it is seen in the Section IV, the regulation capabilities for the MPC controller are really important when the feedback information is delayed or contains errors associated with the selection and errors associated with the measurements. In Figure 3, the scheme including the controller and the estimation system is shown.

\section{Power System Simulation}

In this paper, it is considered that the power system is operating around some values, with random variations of the load (active and reactive) and the power from the renewable generation system, which is, in turn, considered uncontrollable.

The network and microgrid parameters, described in Tables I and II have been used in the simulations. For simulating the power network, a load-flow algorithm computes the voltages and powers from the system, whereas the control system delivers small variations around a defined fixed load value. 


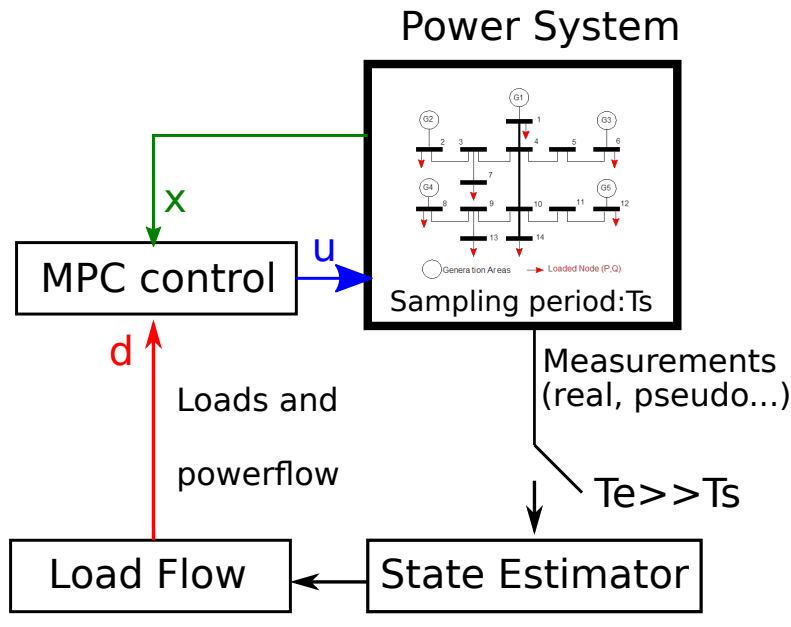

Fig. 3. Controller and estimation blocks structure

A. Initial loads, microgrid states and weighting function values

The following values were considered for the active and reactive loads at the system (zero indicates no load connected):

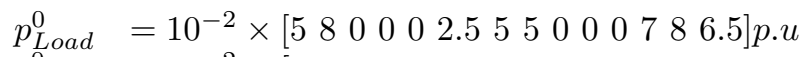

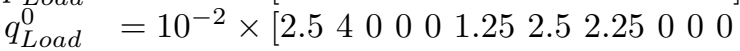

$$
\begin{aligned}
& 3.153 .23 .25] \text { p.u }
\end{aligned}
$$

For the states values at each microgrid, it was considered the following initialization vectors, according to the load flow results for the initial loads (see (1) for better referring each component):

$$
\begin{aligned}
X_{i}^{0} & =\left[\begin{array}{llll}
\theta_{1}^{0} & 50 & 0.5 & 0.9\left(Q_{i}^{0}-Q_{L, i}^{0}\right)
\end{array}\right], \\
U_{i}^{0} & =\left[\begin{array}{lllll}
\left(P_{i}^{0}-P_{L, i}^{0}\right) & 0 & 0 & 0.1\left(Q_{i}^{0}-Q_{L, i}^{0}\right)
\end{array}\right], \\
D_{i}^{0} & =\left[\begin{array}{llll}
P_{L, i}^{0} & P_{i}^{0} & 0 & P_{a l t, i}^{0}\left(Q_{i}^{0}-Q_{L, i}^{0}\right.
\end{array}\right],
\end{aligned}
$$

where the variables with the index "0" are obtained from the initial load flow analysis. Numerical values of (19) are not included for extension reasons. For the controller configuration, it was chosen a prediction horizon $N=2$, with the following diagonal weighting matrices $Q$ and $R$ for each generation area:

$$
\begin{aligned}
& Q=\operatorname{diag}\left\{\left[\begin{array}{lllll}
1 e^{-6} & 80 & 40 & 40 & 25
\end{array}\right]\right\} \\
& R=\operatorname{diag}\left\{\left[\begin{array}{lllll}
0.05 & 0.1 & 0.05 & 0.1
\end{array}\right] .\right.
\end{aligned}
$$

The control objective is related with the following state constraints:

$$
\left[\begin{array}{c}
-2 e^{12} \\
48.5 \\
0.2 \\
0.9 \\
-0.2
\end{array}\right] \leq\left[\begin{array}{c}
\theta_{i} \\
f_{i} \\
S o C_{i} \\
V d c_{i} \\
\delta Q_{i}
\end{array}\right] \leq\left[\begin{array}{c}
2 e^{12} \\
51.5 \\
0.8 \\
1.1 \\
0.2
\end{array}\right]
$$

The objective will be to regulate the frequency, related with the active load compensation and maintain the nodal voltages, related with reactive load compensation. Due to the decentralized structure and the possibility to know the equivalent load transmitted from one microgrid to the rest of the system, the power regulation scheme aids to maintain the full system stability.

\section{B. Measurements set for the state estimation and indirect measurements}

For the proposed structure, it was considered the following measurements:

- Real measurements: voltage magnitude, nodal active and nodal reactive powers for the nodes $1,2,4,6,8,12$ as well as a voltage measurement at node 10 , all of them with an error of $1 \%$.

- Pseudo measurements: active and reactive loads at charged nodes without generators: $\mathbf{7}, \mathbf{1 3}, 14$ with an error of $\mathbf{5 0 \%}$.

- Virtual measurements: null injection at the nodes $3,5,9,10,11$ with an error of $0.001 \%$.

From the state estimator information, the voltage magnitudes and the power flows from two nodes can be obtained as follows [1], [3]:

$$
\begin{aligned}
& \hat{p}_{i j}=\left|\hat{V}_{i}\right|\left|\hat{V}_{j}\right| \cdot\left(g_{i j} \cos \left(\hat{\theta}_{i}-\hat{\theta}_{j}\right)+b_{i j} \sin \left(\hat{\theta}_{i}-\hat{\theta}_{j}\right)\right), \\
& \hat{q}_{i j}=\left|\hat{V}_{i}\right|\left|\hat{V}_{j}\right| \cdot\left(g_{i j} \sin \left(\hat{\theta}_{i}-\hat{\theta}_{j}\right)-b_{i j} \cos \left(\hat{\theta}_{i}-\hat{\theta}_{j}\right)\right) .
\end{aligned}
$$

All the values can be directly replaced in the components of the disturbance vectors, considering only variations around the operative condition (19).

\section{Simulation Results}

Figure 4 shows the behaviour of the states and inputs under active and reactive load and renewable-based generation. The test is limited to $1200 \mathrm{~s}$ and considers the sampling period $T s=0.25 \mathrm{~s}$ and the state estimation each $T e=10 \mathrm{~s}$. The test is focused on showing the performance of the power system under the distributed MPC control, considering process feedback (disturbance knowledge) at a longer sampling rate than the system, which for some closed-loop systems is critical in terms of stability.

Due to space limitations, the Figure 4 shows the frequency, synchronous generators power and battery's SoC for the five microgrids systems. It is shown that power from the generators, the variations of the battery state-of-charge and the frequency are bounded, meaning that active power disturbances are well compensated locally and through the power flows from the generation nodes. This, frequency regulation is achieved.

Also, the reactive power compensation is also well done by the inverters and the output reactance of each generation unit. If the controller would not work as required, the reactive power compensation should increase out of bounds, meaning that voltage stability is not achieved. These kind of analysis are indeed obtained by knowing the power system structure and the power flows, as done in different references such as [1], [2], [4]. 

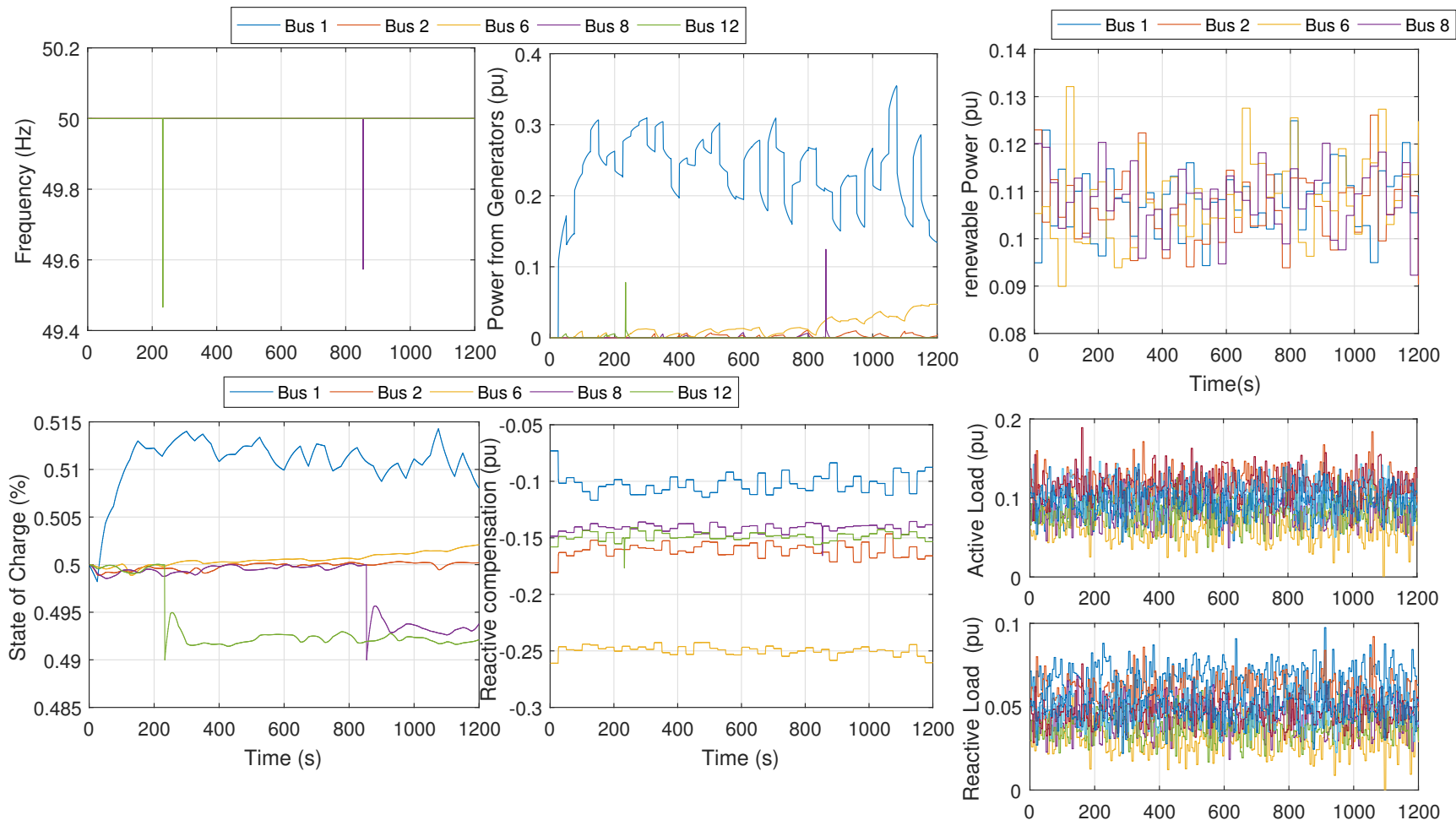

Time (s)

Bus $1-$ Bus $2-$ Bus $6-$ Bus $7-$ Bus $8-$ Bus $12-$ Bus $13-$ Bus 14

Fig. 4. Simulations results for proposed MPC control and estimation

\section{Conclusions And Perspectives}

In this paper, it was tested and verified that a closed-loop control strategy, based on the model predictive methodology is useful for achieving power system stability in distributed generation application, even when the measured data include delays, principally caused by the state estimation methodology. The test was done with noisy disturbances around a defined operation point, from which it is assured the unsaturated state of the power system. In future contributions, the use of this methodology for low-voltage (LV) power systems is proposed, where the lines coupling, unbalanced and intermittent loads, among other factors, make difficult the state estimation and advanced methodologies for this task such as Kalman-like structures should be explored.

\section{REFERENCES}

[1] P. Kundur, N. J. Balu, and M. G. Lauby, Power system stability and control. McGraw-hill New York, 1994, vol. 7.

[2] M. Brenna, E. De Berardinis, L. D. Carpini, F. Foiadelli, P. Paulon, P. Petroni, G. Sapienza, G. Scrosati, and D. Zaninelli, "Automatic distributed voltage control algorithm in smart grids applications," IEEE Transactions on Smart Grid, vol. 4, no. 2, pp. 877-885, 2013.

[3] G. Goodwin, M. M. Seron, and J. A. De Doná, Constrained control and estimation: an optimisation approach. Springer Science \& Business Media, 2006.

[4] J. Sandoval-Moreno, J. J. Martínez, and G. Besançon, "Optimal distributed-coordinated approach for energy management in multisource electric power generation systems," in Developments in Model-Based Optimization and Control. Springer, 2015, pp. 93-114.
[5] A. Ouammi, H. Dagdougui, L. Dessaint, and R. Sacile, "Coordinated model predictive-based power flows control in a cooperative network of smart microgrids," IEEE Transactions on Smart grid, vol. 6, no. 5, pp. 2233-2244, 2015.

[6] J. Alejandro, A. Arce, and C. Bordons, "Combined environmental and economic dispatch of smart grids using distributed model predictive control," International Journal of Electrical Power \& Energy Systems, vol. 54 , pp. 65-76, 2014

[7] A. Abur and A. G. Exposito, Power system state estimation: theory and implementation. CRC press, 2004.

[8] D. Della Giustina, M. Pau, P. A. Pegoraro, F. Ponci, and S. Sulis, "Electrical distribution system state estimation: measurement issues and challenges," IEEE Instrumentation \& Measurement Magazine, vol. 17, no. 6, pp. 36-42, 2014.

[9] IEEE Power Engineering Society, "IEEE 13 Node Test Feeder."

[10] A. Yazdani, A. R. Di Fazio, H. Ghoddami, M. Russo, M. Kazerani, J. Jatskevich, K. Strunz, S. Leva, and J. A. Martinez, "Modeling guidelines and a benchmark for power system simulation studies of three-phase single-stage photovoltaic systems," IEEE Transactions on Power Delivery, vol. 26, no. 2, pp. 1247-1264, 2011.

[11] R. Singh, B. Pal, and R. Jabr, "Choice of estimator for distribution system state estimation," IET generation, transmission \& distribution, vol. 3 , no. 7, pp. 666-678, 2009. 